\title{
EFECTOS SECUNDARIOS DERIVADOS DEL CONSUMO DE ESTEROIDES ANABÓLICOS EN EL DEPORTISTA
}

ADVERSE EFFECTS DERIVED FROM CONSUMPTION OF ANABOLIC STEROIDS IN SPORT

Hernández Fernández, S. ${ }^{1}$, Fernández Salim, M. $^{2}$ y Ortiz González, I. ${ }^{3}$

${ }^{1}$ Graduado en Enfermería por la Universidad de Valladolid. Técnico de Laboratorio de diagnóstico clínico. Enfermero en Sanidad pública de Castilla y León SACYL. España. E-mail: sega32@gmail.com

${ }^{2}$ Doctora en Biología. Profesora en la Escuela Universitaria de Enfermería "Dr. Dacio Crespo" de Palencia, España. E-mail: mfernandez@diputaciondepalencia.es

${ }^{3}$ Graduado en Enfermería por la Universidad de Valladolid. Técnico de laboratorio de diagnóstico clínico. España.

E-mail: onelife522@hotmail.es

\section{FINANCIACION}

El presente artículo no está sujeto financiación externa de ninguna clase más allá de la aportación individual de los autores.

\section{CONFLICTO DE INTERESES}

Los autores declaran que no existe ningún conflicto de intereses.

Código UNESCO: 3207. Patología.

Clasificación del Consejo de Europa: 11. Medicina del deporte.

Recibido el 30 de enero de 2017

Aceptado el 28 de febrero de 2017

Correspondencia:

Hernández Fernández, S: sega32@gmail.com 


\section{RESUMEN}

Los esteroides anabólicos androgénicos son análogos sintéticos químicamente modificados de la testosterona. Desde su descubrimiento, a principios del siglo $\mathrm{XX}$, su uso se ha extendido enormemente en el deporte profesional, y de forma más preocupante, en los adolescentes y adultos jóvenes. Ciertos valores culturales actuales marcan una línea temática que puede desembocar en el consumo de dichas sustancias. El consumo de esteroides anabólicos representa un problema de salud pública. Los efectos secundarios se han documentado extensamente, afectando a multitud de aparatos y sistemas. Dentro de los más relevantes, podemos mencionar los efectos adversos a nivel cardiovascular, psicológico, hepático, reproductivo, neuro-cognitivo, genético y musculo-esquelético. A pesar de los hallazgos evidenciados, muchos de ellos irreversibles, son necesarios nuevos estudios que otorguen mayor conocimiento en la materia.

PALABRAS CLAVE: Esteroides anabólicos androgénicos, anabolizantes, factores de riesgo, efectos secundarios, deporte.

\section{ABSTRACT}

Anabolic androgenic steroids are chemically modified synthetic analogs of testosterone. Since they were discovered, at the beginning of the 20th century, they have been used extensively in professional sports, and more disturbing, in adolescents and young adults. Some current cultural values provide a thematic line that can lead to the consumption of these substances. The consumption of anabolic steroids represents a public health problem. Adverse effects have been extensively documented, affecting a multitude of systems. Among the most relevant, we can mention the adverse effects at cardiovascular, psychological, hepatic, reproductive, neuro-cognitive, genetic and musculo-skeletal. Despite evidenced findings, many of them irreversible, new studies are necessary for greater evidence's matter.

KEY WORDS: Anabolic androgenic steroids, anabolic, risk factors, adverse effects, sport.

\section{INTRODUCCIÓN Y OBJETIVOS}

Los esteroides anabolizantes androgénicos (EAA) son análogos sintéticos químicamente modificados de la testosterona, que incrementan o aumentan los efectos anabolizantes y reducen los efectos androgénicos ${ }^{1,2}$.

En el cuerpo humano, los efectos anabólicos producen un aumento o formación de tejidos, mientras que, los efectos androgénicos son responsables del desarrollo de características sexuales masculinas tanto en hombres como en mujeres ${ }^{1,3}$. 
Los efectos androgénicos y anabolizantes de los EAA son indisolubles y nunca se encuentran disociados, lo que implica que su consumo va a dar lugar a ambos efectos a la vez, sin poder darse uno u otro por separado ${ }^{4,5}$.

Fisiología

Se conocen cinco clases de hormonas esteroideas: estrógenos, andrógenos, progestágenos, mineralocorticoides y glucocorticoides. Los precursores de la vitamina $\mathrm{D}$ también se incluyen por proceder del colesterol aunque poseen diferente estructura ${ }^{6,7}$.

La testosterona y sus derivados, atraviesan fácilmente la membrana celular y se unen a receptores intracelulares específicos, formándose un complejo esteroide-receptor activado que es transportado al núcleo celular, mediante un mecanismo complejo de transducción de señales, dónde estimula la síntesis de proteínas implicadas en los mecanismos de acción de los andrógenos. El catabolismo de la testosterona se produce a nivel hepático, dando lugar a metabolitos excretados por la orina ${ }^{8,9}$.

En el hombre adulto, los testículos producen entre 2,5 y $11 \mathrm{mg} / \mathrm{día}$ de testosterona. Sin embargo, los ovarios de la mujer, y las glándulas suprarrenales producen aproximadamente $0,25 \mathrm{mg} / \mathrm{día}^{9}$.

\section{Marco histórico}

El uso por parte de un deportista de sustancias o métodos prohibidos para progresar en su entrenamiento y mejorar sus resultados deportivos es lo que se conoce como dopaje (doping, en inglés) ${ }^{4,9}$.

El aislamiento de los EAA se logró en 1935 para fines médicos. Pero, no fue hasta los años 1950-59 cuando se tiene constancia del uso de derivados sintéticos de la testosterona, de forma ilícita en el ámbito deportivo por los levantadores de pesas y otros deportistas cuyas disciplinas incluían fuerza, tamaño muscular y resistencia, conceptualizándose de esta forma el llamado "dopaje hormonal" 1, 8, 11-13.

Las primeras actuaciones que incluían controles antidopaje se introducen en los Juegos Olímpicos (JJ.OO.) de Méjico, en 1968, pero no fue hasta los JJ.OO. de Múnich, en 1972, cuando la normativa antidopaje, unida a métodos estandarizados de determinación, experimentaron una imposición contundente ${ }^{11,13}$.

En 1999, por iniciativa del COI se celebra la primera "conferencia mundial sobre el dopaje en el deporte" en Lausana, acordando constituir y poner en funcionamiento la Agencia mundial Antidopaje (AMA) ${ }^{12-14}$.

En el año 2003, la AMA elaboró el "Código Mundial Antidopaje" en el cual se establece la regulación de una serie de normas antidopaje en todos los deportes y países. Uno de los elementos más importantes es la lista de prohibiciones, actualizada anualmente a través de un extenso proceso de 
consulta coordinado por la AMA. La última lista en vigor es válida desde el 1 de enero al 31 de diciembre de $2017^{10,15}$.

\section{Situación en España}

En nuestro país, la Agencia Española de Protección de la Salud en el Deporte (AEPSAD) se encarga de las políticas estatales de protección de la salud y la lucha contra el dopaje en el deporte. Este organismo público asume todas las nuevas competencias que emanan de la Ley Orgánica 3/2013, de 20 de junio de "protección de la salud del deportista y lucha contra el dopaje en la actividad deportiva" ${ }^{16,17}$.

El consumo de esteroides anabólicos androgénicos

En el mundo del deporte olímpico y la alta competición, existe una búsqueda continua de superación atlética. Los deportistas, empujados a menudo por los beneficios sociales y económicos derivados del éxito y bajo la presión a la que se ven sometidos, recurren al uso de sustancias o métodos prohibidos. Una de las sustancias más abundantemente empleadas son los EAA ${ }^{12,18,19}$.

La sociedad contemporánea, marcada por valores como el consumismo, otorga una creciente importancia a la apariencia corporal, esto ha provocado que se invierta más tiempo, energía y recursos económicos en alcanzar o mantener los criterios estéticos en el contexto social. A la vez, existe un incremento del descontento de la autoimagen corporal en la población, constatándose un marcado abuso de EAA en el deporte no profesional con fines estéticos por adolescentes y adultos jóvenes ${ }^{20-23}$.

Los efectos que se buscan con el consumo de EAA son los derivados del potencial anabólico, como la mejora de la composición corporal, aumento del rendimiento deportivo, aumento de la fuerza o mejora de la recuperación ${ }^{24}$.

En líneas generales, la población desconoce que son los EAA y sus efectos secundarios más habituales, tanto reversibles como irreversibles en el organismo. Incluso se cree erróneamente que el abuso de esteroides anabólicos es seguro o sus implicaciones en la salud son mínimas ${ }^{4,26,27}$.

Las principales fuentes de EAA no se someten a controles oficiales, sino que se distribuyen a través de internet, redes sociales inmediatas al usuario (entrenadores, amigos y compañeros de trabajo) y a través de profesionales de la salud (médicos, farmacéuticos o veterinarios) ${ }^{20,28 . ~ A l g u n o s ~ e s t u d i o s ~}$ patrocinados por el COI han demostrado que en España el 13,8\% de los suplementos que se venden a los deportistas pueden contener anabolizantes no incluidos en el etiquetado, los cuales darían dopaje positivo ${ }^{28}$.

Las vías de administración más comúnmente utilizadas para el consumo de esteroides anabólicos son la vía oral e intramuscular, aunque 
también se han descrito el empleo de la vía subcutánea y transdérmica ${ }^{1,4,9,12}$ 30

Existen cientos de EAA diferentes y el patrón habitual de abuso se caracteriza por periodos de consumo seguidos de periodos de descanso. Son los denominados ciclos, cuya duración es muy variable pudiendo durar entre 4 y 12 semanas 1, 9, 12, 29, 31. Estimaciones apuntan a que la dosis de abuso de EAA puede ser entre 10 y 100 veces la dosis terapéutica recomendada, documentándose dosis de entre 250 y $3200 \mathrm{mg}$ a la semana 1, 12, 27, 29, 31 . Además, es habitual el consumo de varios tipos de EAA, practica denominada "stacking" 9, 29. Aunque el efecto anabolizante es dependiente de la dosis, no se ha demostrado cuales son las dosis mínimas necesarias para producir dichos efectos, ni tampoco si el uso de varios EAA a la vez produce un efecto anabólico mayor ${ }^{1}$. Se ha constatado el uso de opiáceos con el objetivo de permitir al individuo seguir entrenando a pesar del dolor muscular y articular, pudiendo resultar como una puerta de entrada para el consumo de otras drogas ${ }^{4,29}$.

Usos terapéuticos

El uso médico de EAA está indicado para el abordaje terapéutico de patologías caracterizadas por una deficiencia endógena de esteroides anabólicos, entre otras aplicaciones.

Son susceptibles de tratamiento con EAA: hipogonadismo y andropausia, hipopituitarismo, osteoporosis, anemias refractarias, carcinoma de mama metastásico (segunda línea de tratamiento), edema angioneurótico, estados catabólicos (desnutrición crónica, caquexia cancerosa, SIDA, etc.), talla baja y depresión entre otros ${ }^{1,9,32,33}$.

\section{Prevalencia}

Se estima que la prevalencia global es del $6,4 \%$ en hombres y $1,6 \%$ en mujeres, que unido al consumo en dosis extremadamente altas y junto a otros medicamentos representan un riesgo para la salud ${ }^{34}$.

Objetivos

Objetivo general

- Definir mediante la revisión documental, los efectos adversos derivados del abuso de esteroides anabólicos androgénicos de forma integral en individuos deportistas.

Objetivos específicos

- Identificar los principales factores de riesgo que precipitan el consumo de esteroides anabólicos. 
- Examinar la prevalencia, población diana y el marco social en el que se sitúa el consumo de los esteroides anabólicos androgénicos en la actualidad.

- Detallar los principios que rigen el consumo y la tendencia evolutiva del problema.

\section{MATERIAL Y MÉTODOS}

La estrategia de búsqueda ha tomado como punto de partida el planteamiento de la pregunta de investigación, siguiendo el modelo PICO (tabla 1).

\section{Tabla 1. Modelo PICO}

\begin{tabular}{|c|c|c|c|}
\hline $\mathbf{P}$ & | & C & 0 \\
\hline $\begin{array}{l}\text { Paciente, problema } \\
\text { o condición }\end{array}$ & Intervención & $\begin{array}{l}\text { Comparación de } \\
\text { la intervención }\end{array}$ & Resultado esperado \\
\hline Deportistas & $\begin{array}{ll}\text { Consumo de } \\
\text { esteroides } & \\
\text { anabólicos } & \end{array}$ & $\begin{array}{l}\text { Deportistas no } \\
\text { consumidores }\end{array}$ & $\begin{array}{l}\text { Efectos } \\
\text { secundarios }\end{array}$ \\
\hline
\end{tabular}

Para la búsqueda documental se ha recurrido a los tesauros DeCS (Descriptores en Ciencias de la Salud) y MeSH (Medical subjets headings). Los términos elegidos se muestran en la tabla 2.

Tabla 2. Descriptores

\begin{tabular}{|l|l|}
\hline DeCS & MeSH \\
\hline Esteroides & Steroids \\
\hline Anabólico, anabolizante, efecto anabolizante & Anabolic agents \\
\hline Efectos adversos, efectos indeseables & Adverse effects \\
\hline Efecto secundario & Secondary Effect \\
\hline Efecto andrógeno & Androgens \\
Uso indebido de drogas en el deporte & Doping in sports \\
\hline Deportes & Sports \\
Atletas & Athletes \\
\hline
\end{tabular}

Criterios de inclusión y exclusión:

Criterios de inclusión

- Artículos en español, inglés o portugués.

- Artículos con al menos tres de los descriptores enunciados. 
- Artículos con acceso gratuito en los que se muestre información concluyente.

\section{Criterios de exclusión}

- Artículos de más de 10 años de antigüedad.

- Ensayos clínicos con animales de laboratorio.

- Artículos con acceso restringido o de suscripción/pago.

- Artículos en otro idioma distinto al español, inglés o portugués.

Resultados de búsqueda:

A continuación se muestran las búsquedas bibliográficas (tabla 3):

\section{Tabla 3. Resultados}

\section{- Medline-PubMed:}

((anabolic agents) OR androgens) AND sports

Filtros: <10 años. Idioma: inglés, español y portugués. Estudios en humanos. Resultados de búsqueda: 1004 artículos. Cumplen criterios: 32 artículos.

(adverse effects AND (anabolic agents OR steroids) AND (sport OR doping in sports))

Filtros: <10 años Idioma: inglés, español y portugués. Estudios en humanos. Resultados de búsqueda: 385 artículos. Cumplen criterios: 16 artículos.

((secondary effects) AND androgens) AND sports

Filtros: <10 años Idioma: inglés, español y portugués. Estudios en humanos. Resultados de búsqueda: 9 artículos. Cumple criterios: 1 artículo.

((adverse effects) AND anabolic agents) AND sports

Filtros: <10 años Idioma: inglés, español y portugués. Estudios en humanos. Resultados de búsqueda: 180 artículos. Cumplen criterios: 23 artículos.

((adverse effects) AND steroids) AND Doping in Sports

Filtros: <10 años Idioma: inglés, español y portugués. Estudios en humanos. Resultados de búsqueda: 148 artículos. Cumplen criterios: 14 artículos.

- BVS (Biblioteca Virtual en Salud): LILACS, IBECS, MedCarib y Medline.

(adverse effects AND steroids) AND sports

Filtros: <10 años. IBECS, LILACS y MedCarib. Idioma inglés, español y portugués. Resultados de búsqueda: 8 artículos. Cumplen criterios: 2 artículos.

((anabolic agents OR steroids) AND (sport)) OR (doping in sports))

Filtros: <10 años. IBECS, LILACS y MedCarib. Idioma inglés, español y portugués. Resultados de búsqueda: 32 artículos. Cumplen criterios: 2 artículos. 


\section{- SciELO}

(anabolic agents OR steroids) AND (adverse effects)

Filtros: < 10 años. Idioma: español inglés y portugués. Resultados de búsqueda: 6 artículos. Cumplen criterios: 3 artículos.

(steroids AND sports)

Filtros: <10 años. Idioma: español inglés y portugués. Resultados de búsqueda: 6 artículos. Cumple criterios: 1 artículo.

(esteroides AND anabólicos)

Filtro: <10 años. Idioma: español inglés y portugués. Resultados de búsqueda: 15 artículos. Cumplen criterios: 4 artículos.

\section{- Elsevier}

(Efectos secundarios de esteroides anabólicos)

Filtros: <10 años. Resultados de búsqueda: 41 artículos. Cumplen criterios: 2 artículos.

(Anabolizantes)

Filtros: <10 años. Resultados de búsqueda: 261 artículos. Cumplen criterios: 4 artículos.

\section{- Google académico}

"efectos secundarios esteroides anabólicos deporte"

Filtros: <10 años, ordenado por relevancia, sin la palabra "tratamiento". Resultados de búsqueda: 78 artículos. Cumplen criterios: 13 artículos.

Análisis de los resultados

Las búsquedas proporcionaron un total 117 referencias que cumplían criterios de inclusión. Tras descartar la bibliografía que se repetía en varias bases bibliográficas, el número total asciende a 65 . Extrayéndose que 32 son revisiones bibliográficas, 25 son estudios observacionales (transversales 0 longitudinales de cohortes, casos y controles, etc.), 1 ensayo clínico y los 7 restantes otros trabajos de investigación.

\section{RESULTADOS Y DISCUSIÓN}

\section{Epidemiología}

Los datos más recientes sitúan la prevalencia mundial del consumo anabolizantes en el $6,4 \%$ de los hombres y el $1,6 \%$ de las mujeres, siendo considerado por una gran cantidad de expertos como un problema de salud pública $3,4,20,21,23,46$. En principio, el uso de EAA se concentró en los deportistas profesionales, donde se ha demostrado una prevalencia de consumo estimada del $9,1 \%$ en deportistas retirados de la liga de futbol americano $^{21}$. 
Actualmente, el uso del EAA ha traspasado las fronteras del deporte profesional para aumentar en los adolescentes y adultos jóvenes que practican deporte de ocio no competitivo o por fines estéticos, situándose la edad media de inicio del consumo entre los 22 y 24 años ${ }^{4}$.

Los mayores porcentajes de prevalencia oscilan entre el $15 \%$ y el $30 \%$, entre aficionados a la musculación en gimnasios y centros de fitness ${ }^{4,47}$. Estos porcentajes son muy variables pudiendo llegar hasta cifras de entre el $29 \%$ y el $67 \%{ }^{48}$. La prevalencia cambia según legislación antidopaje de cada país ${ }^{49}$.

Estudios epidemiológicos realizados en Estados Unidos afirman que entre el $2,7 \%$ y $2,9 \%$ de los adultos jóvenes han tomado EAA al menos una vez en la vida ${ }^{1}$. Otros autores cifran este porcentaje entre el 2,9\% y $4 \%$, lo que supone que entre uno y tres millones de personas han consumido alguna vez dichas sustancias, situando a EE.UU como el país con mayor número de consumidores de EAA ${ }^{4,38}$.

En Europa esta prevalencia se estima en un 2-3\%, siendo cada vez más común, y a edades más tempranas ${ }^{9}$. El proyecto Europeo de Encuestas sobre el Alcohol y otras drogas (ESPAD) sostiene que las cifras en Europa se encuentran entre un $2 \%$ en varones y $1 \%$ en mujeres ${ }^{32}$.

A pesar de los escasos estudios, en nuestro país la extrapolación de los resultados Europeos, sitúan el número de consumidores en 20.000 personas. Según datos de un estudio a cargo de la Generalitat Valenciana en 2006 se estima que un $3,5 \%$ de los jóvenes han consumido alguna vez EAA en España 47,50

En cuanto a los principales factores de riesgo que influyen en el inicio del abuso de EAA se encuentran la existencia previa de mala relación familiar, la preocupación excesiva de la imagen corporal de los individuos, una autoimagen corporal negativa, la baja autoestima, los trastornos de la conducta y los trastornos de la imagen corporal ${ }^{9,23,41,62}$.

Así, algunos autores afirman que la preocupación por la imagen corporal en los consumidores de EAA, para alcanzar el objetivo estético buscado por el individuo, puede desembocar en el abuso de otras drogas coadyuvantes ${ }^{52}$. En consonancia con lo anterior, otros autores sostienen que, de forma inversa, el consumo de otras drogas puede abrir la puerta al consumo de EAA ${ }^{20}$.

Por otro lado, el marco social actual, marcado por valores como el consumismo, individualismo, búsqueda del éxito y culto al cuerpo, lleva al individuo al inicio de la práctica deportiva, y más tarde, al abuso de anabolizantes. Su uso se justifica con la pretensión de conseguir el cuerpo que se desea en un corto periodo de tiempo, venciendo así la insatisfacción individual ${ }^{21}$. Otros autores establecen un inicio simultáneo de la práctica deportiva y el inicio del consumo de EAA ${ }^{51}$. 


\section{Efectos secundarios cardiovasculares}

El consumo de EAA a largo plazo y dosis suprafisiológicas está enormemente vinculado con el desarrollo de patología cardiovascular, dislipemia e hipertensión arterial ${ }^{1}$.

Los esteroides anabólicos, están implicados en fenómenos aterogénicos, trombóticos y vasoespásticos a nivel intracardiaco y periférico ${ }^{53}$. Se ha demostrado que provocan una lesión miocárdica directa a nivel celular y su administración crónica provoca una reducción de la sensibilidad barorrefleja debida a la degeneración de las neuronas simpáticas endomiocárdicas ${ }^{4,53-55}$.

El evento más destacado, es el deterioro de la función contráctil del miocardio, con la consecuente reducción del volumen de eyección del ventrículo izquierdo ${ }^{4,55}$. También se produce una disminución de la elasticidad aortica $4,56,57$. Estos cambios pueden ser parcialmente reversibles si se suspende el consumo, no ocurre lo mismo con el aumento de la fibrosis miocárdica y por tanto la perdida de elasticidad, que es de carácter permanente 52, 56. Asimismo la cardiotoxicidad asociada al consumo de EAA es impredecible y está relacionada directamente con la dosis y el tiempo de abuso ${ }^{57}$.

También se ha determinado que el consumo de EAA está relacionado con una reducción patológica de la función diastólica. El llenado diastólico final es menor y se produce de forma más tardía $55,56,58,59$. La literatura también describe una reducción de la función sistólica del ventrículo izquierdo, alteraciones repolarización ventricular y cambios en la regulación autónoma del corazón ${ }^{4}$.

Así mismo, también se ha observado que el consumo de EAA unido a la práctica deportiva condiciona la aparición de hipertrofia del ventrículo izquierdo $(\mathrm{HVI})^{52,59}$. Sin embargo, otros hallazgos coinciden en mayor medida en que el desarrollo de la HVI concéntrica es una respuesta fisiológica adaptativa, y solo un pequeño porcentaje sufre hipertrofia excéntrica patológica ${ }^{53,55}$. En conclusión, la evidencia clínica disponible es diversa, no obstante, en concordancia con la discusión de varios autores, se puede afirmar que el consumo de EAA parece no producir de forma sistemática HVI y que ésta, se origina con el tiempo a consecuencia del ejercicio de carácter anaeróbico ${ }^{55-57}$.

Otros estudios aseveran que los EAA prolongan caóticamente la repolarización, aumentando de esta forma el riesgo de sufrir arritmias graves ${ }^{53}$. Las más comúnmente documentadas son; la fibrilación auricular, la fibrilación ventricular, taquicardia ventricular y supraventricular y extrasístoles ventriculares $^{48}$.

Entre las complicaciones más frecuentes se encuentran la hipertensión, la insuficiencia cardiaca, la miocardiopatía dilatada, el infarto agudo de miocardio (IAM), la enfermedad coronaria, y la muerte súbita ${ }^{53}$. Debido a que ciertas características el deterioro miocárdico son irreversibles, se espera que 
en los próximos años haya un aumento de la tasa de eventos cardiovasculares graves en individuos que abandonaron el abuso de EAA en la juventud ${ }^{52}$. Es importante resolver que aunque los últimos estudios confirman la aparición de hallazgos patológicos, otros autores no han encontrado relación etiopatológica entre el consumo de EAA y los eventos cardiacos ${ }^{55}$.

Por otro lado, el perfil lipídico se ve alterado, produciéndose un aumento del colesterol total, las LDL, y una disminución de las HDL ${ }^{4,58}$. Varios autores atribuyen los cambios en el perfil lipídico a un aumento de la actividad de la lipoproteína lipasa, la principal enzima responsable del catabolismo de las HDL 59. Los EAA determinan un estado de hipercoagulabilidad y riesgo trombótico al promover una mayor agregación plaquetaria, producción de tromboxano a2, disminución de la producción de prostaglandina 12 y aumento de los niveles de fibrinógeno. Dicho estado puede aumentar el riesgo de enfermedad coronaria entre 3 y 6 veces ${ }^{48}$. Se estima que las LDL pueden aumentar más del $20 \%$ y disminuir las HDL entre un $20 \%$ y un $70 \%$, siendo estos cambios objetivables dentro de un periodo de 9 semanas tras el inicio del consumo y normalizarse tras 5 meses.

Por último, se produce una retención de sodio en el organismo que provoca habitualmente retención hídrica, incrementándose el volumen plasmático hasta en un $15 \%$ e instaurándose de ésta forma un aumento de la presión arterial ${ }^{9}$.

Efectos secundarios hepáticos

A nivel hepático, se ha demostrado que el consumo de EAA (principalmente las preparaciones orales) a dosis suprafisiológicas provocan hepatotoxicidad y son responsables de un aumento de las enzimas alanina aminotransferasa (ALT) y aspartato aminotransferasa (AST). Se tiene constancia de que, tras cesar el consumo, se produce una mejora de la función hepática $47,52,59$.

Aunque algunos autores afirman que hay escasa evidencia de disfunción hepática en varias pruebas de laboratorio y exámenes físicos, estudios in vitro han concluido que dosis suprafisiológicas causan apoptosis celular ${ }^{52}$. También se han documentado casos de hepatomegalia, peliosis hepática y colestasis intrahepática, angiosarcoma hepático, colangiosarcoma, adenoma y carcinoma hepatocelular y ruptura hepática espontanea ${ }^{1,47,52,59,60}$.

\section{Efectos secundarios neurológicos y psicológicos}

Está ampliamente aceptado que los esteroides anabólicos provocan cambios psicológicos sustanciales $1,4,9,55,61$. El consumo no controlado de EAA, puede desencadenar síntomas maniacos e hipomaniacos que a menudo se manifiestan como conductas agresivas e irritabilidad, incluso en individuos sin antecedentes previos. Es habitual la aparición de un aumento de la energía, 
sensación de euforia, aumento de la libido, confusión y distracción. Estos síntomas pueden ser percibidos como positivos por el individuo ${ }^{1,4,60}$.

Tras la abstinencia, es común la aparición de síntomas depresivos caracterizados por astenia, anorexia, perdida de interés por actividades habituales, perdida de la libido, hipersomnia e incluso tendencias suicidas ${ }^{1,4,9}$, 52, 60. La aparición de los síntomas descritos está sujeta a factores de vulnerabilidad individual, la mayoría de los usuarios solo experimenta alguno de los síntomas y rara vez se presentan los más graves e incapacitantes. La mayoría de los estudios describe cambios anímicos a corto plazo cuya duración puede establecerse en semanas o meses ${ }^{52}$. Es importante destacar que los efectos adversos a nivel psicológico, son más habituales a dosis altas, de más de $1000 \mathrm{mg} /$ semana $^{4}$.

La dependencia también es un efecto secundario al consumo de EAA ${ }^{1}$, 4, 9, 60. Aparece aproximadamente en el $30 \%$ de los individuos $4,62,63$. A diferencia de otras sustancias, los esteroides anabólicos no ofrecen un efecto de recompensa inmediato tras su administración por lo que la dependencia gira entorno a otros mecanismos.

La existencia previa de trastornos de la imagen corporal pueden llevar al individuo a desarrollar dependencia como consecuencia de la ansiedad que supone dejar de consumir EAA y ver cambiada la imagen corporal ${ }^{4,62}$. Sin embargo, datos de un estudio poblacional, concluyeron que aunque la preocupación por la imagen puede desencadenar el consumo de EAA, aquellos individuos que generaron dependencia no mostraban mayor preocupación por la imagen corporal que los que no. En conclusión, podemos extraer que aparentemente no hay relación directa entre el grado de preocupación por la imagen y la dependencia ${ }^{62}$.

El sistema neuroendocrino también es una pieza clave en el síndrome de dependencia a dichas sustancias. La administración exógena de EAA altera el funcionamiento normal del eje hipotalámico-hipofisario-testicular, pudiendo causar hipogonadismo de duración condicionada al restablecimiento de los niveles de testosterona endógena, la cual puede tardar meses, años o incluso no restablecerse en algunos casos. Los síntomas derivados del hipogonadismo pueden conducir al consumo de otras sustancias como el clomifeno o la gonadotropina coriónica humana ( $\mathrm{hCG}$ ) para paliar esta situación y reanudar más tarde el consumo de esteroides ${ }^{4,55}$.

La dependencia a los EAA comparte características con la dependencia a los opioides. Los anabólicos podrían potenciar la actividad opioide endógena central, que tras el cese del consumo daría lugar a una disminución de dicha actividad y a un posterior síndrome hiperadrenérgico agudo. Las drogas clásicas ofrecen una recompensa rápida generada por la intoxicación aguda, principal diferencia con los anabólicos que cuentan con un mecanismo de recompensa de aparición tardía ${ }^{62}$. 
En la última década, las investigaciones relacionan las dosis suprafisiológicas de esteroides con la neurotoxicidad 4, 52, 63. Un estudio realizado en Inglaterra reveló que los consumidores de esteroides anabólicos mostraban déficits sustanciales y estadísticamente significativos en competencias cognitivas. Por tanto, se extrae que los EAA pueden causar neurotoxicidad en grado variable. A pesar de las afirmaciones anteriores, no hay constancia de ningún caso de patología neurodegenerativa. En línea con la discusión del autor, una hipótesis que puede explicar la situación se basa en que la población diana es demasiado joven para manifestar los efectos neurotóxicos y déficits cognitivos ya que en su gran mayoría, la población expuesta es menor de 50 años ${ }^{63}$. La evidencia clínica disponible acerca de las implicaciones psicológicas del consumo de anabólicos ha de interpretarse con cautela $^{4,62}$.

\section{Efectos secundarios reproductivos y endocrinos}

En el varón, se ha informado de la aparición de azoospermia u oligospermia, se estima que la oligospermia se da en un $75 \%$ de los individuos 9. La atrofia testicular aparece en el $40-51 \%$ de los casos y la ginecomastia en el $10-34 \% 59$. La interrupción de la espermatogénesis en el hombre es de carácter reversible, pudiendo restablecerse en una media de 6,3 meses ${ }^{4,59}$. Una revisión del 2015 expone que en el $67 \%$ de los hombres el recuento espermático alcanzó los 20 millones $/ \mathrm{ml}$ en 6 meses, el $90 \%$ en 12 meses, el $96 \%$ en 16 meses y el $100 \%$ en 24 meses ${ }^{4}$.

A nivel clínico, los pacientes pueden presentar un patrón seminológico de azoospermia, con un volumen seminal normalmente inalterado ${ }^{47}$. Por otro lado, la masculinización en las mujeres puede revestir permanencia aun con la supresión del consumo de anabólicos ${ }^{9,59}$.

La administración exógena de EAA induce cambios en el eje hipotalámico-hipofisario-testicular. La falta de andrógenos endógenos provoca la pérdida gradual de la capacidad madurativa de espermatozoides del epidídimo, desencadenando atrofia testicular que puede ser de hasta el $20 \%$. Se instaura de esta forma un cuadro de hipogonadismo gonadotrópico inducido por esteroides (ASIH) 1, 4, 9, 47. Algunos autores afirman que los niveles de LH y $\mathrm{FSH}$ son directamente proporcionales al número de espermatozoides ${ }^{4}$. En ocasiones se recurre a otras sustancias para evitar la atrofia testicular, comúnmente a la hCG pero su incapacidad para estimular la FSH y así la maduración espermática, concede el fracaso ${ }^{9}$.

En la mujer, la supresión del eje hipotalámico-hipofisario-gonadal desencadena disminuciones de la $\mathrm{LH}$, la $\mathrm{FSH}$, los estrógenos y la progesterona. En contraposición, algunas investigaciones afirman que disminuyen los niveles de $\mathrm{FSH}$ y las concentraciones de la globulina transportadora de hormonas sexuales (SHBG) pero no estiman cambios en la $\mathrm{LH}^{1}$. 
Sobre los efectos en el ciclo menstrual, habitualmente provoca dismenorrea o amenorrea con anovulación ${ }^{4}$. Otras investigaciones exponen que el ciclo menstrual sigue produciéndose con un incremento de la fase folicular y el acortamiento de la fase lútea ${ }^{59}$.

En adolescentes puede precipitar el cierre prematuro de las epífisis óseas y por lo tanto detener el crecimiento, obteniendo una talla inferior a la normal y una maduración precoz del aparato reproductor ${ }^{9}$.

\section{Efectos secundarios musculo-esqueléticos}

En el tejido muscular, la administración de EAA tiene efectos tróficos, promueven la hipertrofia muscular de las fibras de forma individual, sin aumento en el número de fibras.

Se ha demostrado que los EAA fomentan cambios en el metabolismo muscular de forma indirecta. Los receptores de glucocorticoides se relacionan con el catabolismo proteico. Así pues, la unión de los anabólicos a dichos receptores origina un bloqueo del catabolismo proteico a nivel muscular, explicando el posterior crecimiento muscular ${ }^{54}$. El consumo se vincula con lesiones en las estructuras tendinosas, según algunos estudios, la patogénesis se atribuye a cambios adaptativos rápidos en la fuerza por acción hormonal que no se producen del mismo modo en los tendones menos vascularizados, provocando roturas ${ }^{1,4,54}$.

Los datos extraídos de un estudio sobre jugadores de futbol americano revelaron que aquellos consumidores habituales presentaban mayores lesiones articulares, cartilaginosas y meniscales que los no consumidores ${ }^{59}$.

\section{Efectos secundarios genotóxicos y cáncer}

Los andrógenos a menudo se consideran promotores del carcinoma de próstata por lo que se podría esperar una tasa alta de hipertrofia benigna de próstata y cáncer de próstata ${ }^{4}$. A pesar de dicha afirmación, la evidencia solo recoge casos aislados como un caso leiomiosarcoma intratesticular y un caso de cáncer de próstata ${ }^{1}$.

La mayor parte de la bibliografía coincide en que un aumento de la testosterona sérica, no promueve un mayor riesgo de aparición de cáncer en ambos sexos. En contraste, otras investigaciones exponen que en las mujeres premenopaúsicas se desencadena un pequeño aumento del riesgo de desarrollo de ciertos carcinomas correlacionados con los niveles de testosterona y androstenediona séricos ${ }^{4}$.

En conclusión, la evidencia disponible actualmente sobre el cáncer y los EAA es limitada. Predominan las estimaciones que niegan una relación entre el consumo y la carcinogénesis, más relacionada con factores individuales ${ }^{4,52}$. 


\section{Otros efectos secundarios}

En ambos sexos encontramos el acné vulgaris, la piel grasa, las estrías, el hirsutismo y la alopecia de patrón masculino. Algunos estudios demuestran que entre el $17 \%$ y el $50 \%$ sufrieron acné, y más del $40 \%$ estrías ${ }^{4}$, mientras que otros autores estiman porcentajes similares, de entre el $40-54 \%$ el acné y el $34 \%$ las estrías ${ }^{59}$. Dichos efectos secundarios son reversibles, en el caso del acné grave es posible que queden cicatrices y en el caso de las estrías, pueden persistir como rayas blancas ${ }^{1,4,59}$.

La reutilización e intercambio de agujas puede provocar la transmisión de agentes infecciosos como el $\mathrm{VIH}$, la hepatitis $\mathrm{B}$ o la hepatitis $\mathrm{C} O$ Staphylococcus aureus ${ }^{4}$.

Por otro lado, existe una fuerte controversia documental sobre las posibles repercusiones clínicas de los esteroides en la regulación de la glándula tiroides. Se ha observado que el consumo a dosis altas de EAA desencadena una disminución de la globulina fijadora de tiroxina (TBG) y de los niveles de T3 y T4, pudiendo actuar como factores de confusión con cuadros de hipotiroidismo subclínico. Por lo tanto el análisis de los resultados lleva a pensar que se establece una relación aun poco reveladora entre el consumo de anabólicos y la patología tiroidea $^{38}$.

\section{REFERENCIAS BIBLIOGRÁFICAS}

1. Lafranco F, Ghigo E, Strasburger CJ. Hormonas y actividad física. En: Kronenberg HM, Melmed S, Polonsky KS, Reed Larsen P. Williams Tratado de

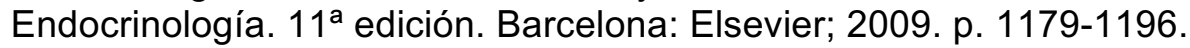

2. García C, Hervás JV, Jiménez M, Pérez de la Ossa N, Gomis M, Dorado L, et al. Ingesta de esteroides anabolizantes e ictus isquémico. Presentación de un caso clínico y revisión de la bibliografía. Rev Neurol 2013; 56: 327-31.

3. National Institute on Drug Abuse (NIH) [Internet]. Maryland: NIDA; 2007 [Acceso 19 de diciembre 2015]. ¿Qué son los esteroides anabólicos androgénicos? [aprox. 2 p.]. Disponible en: https://www.drugabuse.gov/es/publicaciones/serie-de-reportes/abuso-de-losesteroides-anabolicos/que-son-los-esteroides-anabolicos

4. Pope HG, Wood RI, Rogol A, Nyberg F, Bowers L, Bhasin S. Adverse health consequences of performance-enhancing drugs: an Endocrine Society scientific statement. Endocr Rev. 2014 Jun; 35: 341-75.

5. Rodríguez A, Rosenberg L, Moscona G, Gabba M, Mendonça M, Martínez C. Etnografía de usuarios de drogas esteroides anabólicas en gimnasios de la Ciudad de Buenos Aires. Resultados preliminares. En: V Jornadas de Sociología de la Universidad Nacional de La Plata (UNLP). La Plata: Departamento de Sociología; 2008. p. 1-19.

6. Hormonas [Internet]. Elche: Miguel Hernández Universitas; 2013 [Acceso 26 de diciembre 2015]. Colesterol III hormonas esteroideas [aprox. 5 pantallas]. Disponible en: http://las-hormonas.blogspot.com.es/2013/09/colesterolhormonas-esteroideas.html

7. Biología y salud [Internet]. Madrid: Ministerio de Ciencia y Tecnología; 2010. [Acceso 4 de enero 2016]. Hormonas esteroides [aprox. 3 pantallas]. 
Disponible

en:

http://www.biopsicologia.net/Nivel3participaci\%C3\%B3npl\%C3\%A1sticayfuncional/7-hormonas-esteroides

8. Orrero JJ. Eje hipotálamo hipófisis. En: Sierra DM, director. Fundamentos de medicina y endocrinología. $7^{\text {a }}$ edición. Colombia: Corporación para Investigaciones Biomédicas (CIB); 2012. p. 13-38.

9. Barrientos M. Uso y abuso de anabólicos esteroideos (AE). En: Mendoza C, editor. Endocrinología clínica. $4^{a}$ edición. México: Manual Moderno; 2012. p. 853-859.

10. Álvarez C, Cortés N, Espinosa P, Fernández L, Fernández C, Frutos $A M$, et al. Historia del dopaje, sustancias y procedimientos de control Vol. I [Monografía en internet]. $1^{\text {a }}$ edición. Madrid: Consejo superior de deportes; 2008 [Acceso 7 de enero 2016]. Disponible en http://www.csd.gob.es/csd/estaticos/documentos/52_150.pdf

11. Burgueño R, López D, García A. El dopaje en el deporte: reseña histórica. Rev Dig Buenos Aires [Revista en internet]. 2012 [Acceso 13 de enero 2016]; 17(168):1-25. Disponible en: http://www.efdeportes.com/efd168/el-dopaje-en-eldeporte-resena-historica.htm

12. Laudo C, Puigdevall V, del Río MJ, Velasco A. Hormonas utilizadas como agentes ergogénicos: situación actual del problema. An Sist Sanit Navar [revista en internet]. 2006 [Acceso 23 de febrero 2016]; vol. 29 (2): 207-218. Disponible en: http://scielo.isciii.es/scielo.php?pid=S113766272006000300005\&script=sci_arttext

13. Handelsman DJ. Performance Enhancing Hormone Doping in Sport. In: De Groot LJ, Beck P, Chrousos G, editors. Endotext [Internet]. South Dartmouth (MA): MDText; 2000. [Acceso 24 de enero 2016]. Disponible en: http://www.ncbi.nlm.nih.gov/books/NBK305894/

14. Ramos A, Ruiz J, Quiroga M. Dopaje y Aparato Cardiovascular: efectos y alteraciones. Canarias Médica y Quirúrgica [Internet]. 2008 [Acceso 24 de febrero 2016]; Vol. 6 (17): [32-39]. Disponible en: http://acceda.ulpgc.es/bitstream/10553/5958/1/0514198_00017_0006.pdf

15. Agencia Mundial Antidopaje (AMA) [Internet]. Canadá: WADA - AMA; 2016 [Acceso 25 de febrero 2017]. Disponible en: https://www.wadaama.org/sites/default/files/resources/files/2016-10-

28_wada_prohibited_list_2017_sp_final.pdf

16. Ministerio de Educación, Cultura y Deporte [internet]. Madrid: Agencia Española de Protección de la Salud en el Deporte (AEPSAD); 2015 [Acceso 26 de febrero 2016]. La agencia [1]. Disponible en: http://www.mecd.gob.es/aepsad/agencia.html

17. Ley Orgánica $3 / 2013$, de 20 de junio, de protección de la salud del deportista y lucha contra el dopaje en la actividad deportiva. (Boletín Oficial del Estado, núm. 148 de 21 de junio de 2013).

18. Atienza E. Dopaje y enfermedad mental: más allá de la responsabilidad del deportista [Internet]. Universidad de Deusto: País Vasco; 2012 [Acceso 27 de enero 2016]. Disponible en: http://www.academia.edu/5734497/Dopaje_y_enfermedad_mental_m\%C3\%A1 s_all\%C3\%A1_de_la_responsabilidad_del_deportista

19. Madrid M, Ramírez R, Rigioni C, Vargas E. Ayudas ergogénicas. En: Fisiología Avanzada del Ejercicio. Costa Rica: Facultad Nacional de Ciencias de la Salud; 2006. p. 1-30. 
20. Sagoe D, Andreassen CS, Pallesen S. The aetiology and trajectory of anabolicandrogenic steroid use initiation: a systematic review and synthesis of qualitative research. Subst Abuse Treat Prev Policy [Internet]. 2014 [Acceso 27 de febrero 2016]; 2(9). Disponible http://www.ncbi.nlm.nih.gov/pmc/articles/PMC4091955/

21. Iriart JA, Chaves JC, Orleans RG. Body cult and use of anabolic steroids by bodybuilders. Cad Saude Pública [Revista en internet]. Abril 2009 [Acceso 28 de febrero 2016]; 25(4):773-82. Disponible en: http://www.ncbi.nlm.nih.gov/pubmed/19347203

22. Melnik BC. Androgen abuse in the community. Curr Opin Endocrinol Diabetes Obes [Revista en internet]. Junio 2009 [Acceso 26 de febrero 2016]; 16(3):21823. Disponible en: http://www.ncbi.nlm.nih.gov/pubmed/19373082

23. Jenssen $\mathrm{IH}$, Johannessen KB. Aggression and body image concerns among anabolic androgenic steroid users, contemplators, and controls in Norway. Body Image [Internet]. Enero 2015 [Acceso 4 de febrero 2016]; 12:6-13. Disponible en: http://www.ncbi.nlm.nih.gov/pubmed/25261635

24. Yu JG, Bonnerud $P$, Eriksson A, Stål PS, Tegner $Y$, Malm C. Effects of long term supplementation of anabolic androgen steroids on human skeletal muscle. PLoS One [Internet]. Septiembre 2014 [Acceso 27 de febrero 2016]; 9(9):e105330.

Disponible http://www.ncbi.nlm.nih.gov/pmc/articles/PMC4160183/

25. Domínguez EB, Fernández PN, Giménez JF, Gerometta R. Estudio descriptivo del consumo de esteroides anabólicos en la población que asiste a gimnasios de la Ciudad de Corrientes. Postgrado VI cátedra Med. Abril 2013; 1(214): 219.

26. Hernández I, Hernández R, Ramos M, Santana A. Fármacos dopantes. Rev farm Esp. 2013 1(12): 3-37.

27. Irigoyen-Coll C. Esteroides y percepción del riesgo. Salud y drogas [Revista en internet]. 2005 [Acceso 7 de marzo 2016]; 5(1):119-137. Disponible en: http://www.redalyc.org/articulo.oa?id=83950107

28. Casajús JA. Dopaje, salud y deporte. Información terapéutica del Sistema Nacional de Salud [Revista en internet]. 2005 [Acceso 4 de marzo 2016]; 29(1):1-11. Disponible en: http://www.msssi.gob.es/biblioPublic/publicaciones/docs/vol29_1dopaje.pdf

29. Suárez MP. Anabolizantes. Complicaciones cardiovasculares. Muerte súbita inesperada. Cuadernos de Medicina Forense [Revista en internet]. 2009 [Acceso 24 de enero 2016]; 1(26):29-39. Disponible en: http://scielo.isciii.es/pdf/cmf/n26/original4.pdf

30. Avella RE, Medellín JP. Los esteroides anabolizantes androgénicos, riesgos y consecuencias. Rev. U.D.C.A Act. \& Div. Cient [Revista en internet]. 2012 [Acceso 12 de enero 2016]; 15 (Supl. Olimpismo):47-55. Disponible en: http://www.scielo.org.co/pdf/rudca/v15s1/v15s1a07.pdf

31. Asensio VM. Esteroides anabolizantes: una visión de conjunto. Semergen [Revista en internet]. 2012 [Acceso 28 de enero 2016]; 28(5):245-48. Disponible en: http://www.elsevier.es/en-revista-semergen-medicina-familia-40articulo-esteroides-anabolizantes-una-vision-conjunto-13032571

32. Kersey RD, Elliot DL, Goldberg L, Kanayama G, Leone JE, Pavlovich M, et al. National Athletic Trainers' Association. National Athletic Trainers' Association position statement: anabolic-androgenic steroids. J Athl Train [Internet]. 
Septiembre 2012 [Acceso 14 de marzo 2016]; 47(5):567-588. Disponible en: http://www.ncbi.nlm.nih.gov/pmc/articles/PMC3465038/

33. Paiva M. Guía sobre medicamentos y sustancias de abuso: descripción y efectos. En: Comité de psicología. Medellín: Universidad de San Buenaventura Seccional; 2013. p. 1-73.

34. Nieschlag E, Vorona E. Mechanisms in endocrinology: Medical consequences of doping with anabolic androgenic steroids: effects on reproductive functions. Eur J Endocrinol [Internet]. Agosto 2015 [Acceso 4 de febrero 2016]; 173(2):R47-58. Disponible en: http://www.ejeonline.org/cgi/pmidlookup?view=long\&pmid=25805894

35. Hallberg M. Impact of anabolic androgenic steroids on neuropeptide systems. Mini Rev Med Chem [Internet]. Mayo 2011 [Acceso 5 de marzo 2016]; 11(5):399-408. Disponible en: http://www.ncbi.nlm.nih.gov/pubmed/21443512

36. Razavi Z, Moeini B, Shafiei Y, Bazmamoun H. Prevalence of anabolic steroid use and associated factors among body-builders in Hamadan, West province of Iran. J Res Health Sci [Internet]. Abril 2014 [Acceso 15 de marzo 2016]; 14(2):163-6. Disponible en: http://www.ncbi.nlm.nih.gov/pubmed/21443512

37. Kanayama G, Kean J, Hudson JI, Pope HG Jr. Cognitive deficits in long-term anabolic-androgenic steroid users. Drug Alcohol Depend [Internet] 2013 junio. [Acceso 18 de marzo 2016]; 130(1-3):208-14. Disponible en: http://www.ncbi.nlm.nih.gov/pmc/articles/PMC3608708/

38. Fortunato RS, Rosenthal D, Carvalho DP. Abuse of anabolic steroids and its impact on thyroid function. Arq Bras Endocrinol Metabol [Internet]. Diciembre 2007 [Acceso 21 de marzo 2016]; 51(9):1417-24. Disponible en: http://www.ncbi.nlm.nih.gov/pmc/articles/PMC3608708/

39. Dorn SB, Bolt HM, Thevis M, Diel P, Degen GH. Micronucleus induction in V79 cells by the anabolic doping steroids desoxymethyltestosterone (madol) and 19norandrostenedione. Toxicol Lett [Internet]. Diciembre 2008 [Acceso 2 de marzo 2016]; 183(1-3):58-64. Disponible en: http://onlinelibrary.wiley.com/doi/10.1038/bjp.2008.165/full

40. Wollina U, Pabst F, Schönlebe J, Abdel-Naser MB, Konrad H, Gruner M, et al. Side-effects of topical androgenic and anabolic substances and steroids. A short review. Acta Dermatovenerol Alp Pannonica Adriat [Internet]. Septiembre 2007 [Acceso 2 de abril 2016]; 16(3):117-22. Disponible en: http://s3-eu-west1.amazonaws.com/thejournalhub/10.15570/archive/acta-apa-07-3/5.pdf

41. Pope HG Jr, Kanayama G, Hudson JI. Risk factors for illicit anabolic-androgenic steroid use in male weightlifters: a cross-sectional cohort study. Biol Psychiatry [Internet]. Febrero 2012 [Acceso 22 de marzo 2016]; 71(3):254-61. Disponible en: http://www.ncbi.nlm.nih.gov/pmc/articles/PMC3218214/

42. Pinto F, Sacco E, Volpe A, Gardi M, Totaro A, Calarco A, et al. Doping and urologic tumors. Urology [Internet]. Abril-mayo 2010 [Acceso 22 de febrero 2016]; 77(2):92-9. Disponible en: http://www.ncbi.nlm.nih.gov/pubmed/20890867

43. Carmo EC, Fernandes T, Oliveira, EM. Esteroides anabolizantes: La cardiopatía en el atleta a causa de los esteroides anabólicos. Rev. educ. fis [Internet]. 2012 [Acceso 2 de abril 2016]; 23(2): 307-318. Disponible en: http://pesquisa.bvsalud.org/portal/resource/es/lil-701456

44. Chulvi I, Pomar A, Pomar R. Un problema de salud pública: uso de esteroides anabólicos en los centros fitness. Rev Dig Buenos Aires [Revista en internet]. Enero 2007 [Acceso 2 de abril de 2016]; 11(104): 1-13. Disponible en: 
http://www.efdeportes.com/efd104/uso-de-esteroides-anabolicos-en-loscentros-fitness.htm

45. Ritter JM. Sex, steroids and anabolic androgens in athletics. $\mathrm{Br} \mathrm{J}$ Clin Pharmacol [Internet]. Julio 2012 [Acceso 3 de abril 2016]; 74(1):1-2. Disponible en: http://www.ncbi.nlm.nih.gov/pmc/articles/PMC3394123/

46. Arbinaga F. Consumo de esteroides anabolizantes en el fisioculturismo; relaciones con variables del entrenamiento y la imagen corporal. Rev Iber psic ejerc y dep [Revista en internet]. 2008 [Acceso 6 de abril 2016]; 3(1):47-60. Disponible en: http://dialnet.unirioja.es/servlet/articulo?codigo $=2720013$

47. García F. Implicaciones andrológicas del abuso de esteroides androgénicos anabolizantes. Rev int Androl [Revista en internet]. Octubre 2011 [Acceso 8 de abril 2016]; 9(4): 160-169. Disponible en: http://apps.elsevier.es/watermark/ctl_servlet?_f=10\&pident_articulo=90063665 \&pident_usuario $=0 \&$ pcontactid $=\&$ pident_revista $=262 \&$ ty $=17$ \&accion=L\&origen $=$ zonadelectura\&web=www.elsevier.es\&lan $=e s \&$ fichero $=262 v 09 n 04 a 90063665 p$ df001.pdf

48. Achar S, Rostamian A, Narayan SM. Cardiac and metabolic effects of anabolicandrogenic steroid abuse on lipids, blood pressure, left ventricular dimensions, and rhythm. Am J Cardiol [Internet]. Septiembre 2010 [Acceso 7 de abril 2016]; 106(6):893-901. Disponible

en: http://www.ncbi.nlm.nih.gov/pmc/articles/PMC4111565/

49. Leifman H, Rehnman C, Sjöblom E, Holgersson S. Anabolic androgenic steroids -use and correlates among gym users- an assessment study using questionnaires and observations at gyms in the Stockholm region. Int $\mathrm{J}$ Environ Res Public Health [Internet]. Julio 2011 [Acceso 13 de abril 2016]; 8(7):265674. Disponible en: http://www.injuryjournal.com/article/S0020-1383(11)001136/abstract

50. Torrijo MJ. Encuesta escolar de la Comunidad Valenciana. Fundación para el Estudio, Prevención y Asistencia a Drogodependencias (FEPAD). 2006. p. 113.

51. Kanayama G, Hudson JI, Pope HG Jr. Features of men with anabolicandrogenic steroid dependence: A comparison with nondependent AAS users and with AAS nonusers. Drug Alcohol Depend [Internet]. Junio 2009 [Acceso 18 de abril 2016]; 102(1-3):130-7. Disponible en: http://www.ncbi.nlm.nih.gov/pmc/articles/PMC2694450/

52. Kanayama G, Brower KJ, Wood RI, Hudson JI, Pope HG Jr. Anabolicandrogenic steroid dependence: an emerging disorder. Addiction [Internet]. Diciembre 2009 [Acceso 3 de mayo 2016]; 104(12):1966-78. Disponible en: http://www.ncbi.nlm.nih.gov/pmc/articles/PMC2646607/

53. Deligiannis AP, Kouidi El. Cardiovascular adverse effects of doping in sports. Hellenic J Cardiol [Internet]. Noviembre-diciembre 2012 [Acceso 18 de mayo 2016]; 53(6):447-57. Disponible en: http://www.hellenicjcardiol.org/archive/full_text/2012/6/2012_6_447.pdf

54. Stergiopoulos K, Brennan JJ, Mathews R, Setaro JF, Kort S. Anabolic steroids, acute myocardial infarction and polycythemia: a case report and review of the literature. Vasc Health Risk Manag [Internet]. 2008 [Acceso 18 de mayo 2016]; 4(6):1475-80. Disponible en: https://www.dovepress.com/articles.php?article_id=2602

55. D'Andrea A, Caso P, Salerno G, Scarafile R, De Corato G, Mita C, Di Salvo G, Severino S, Cuomo S, Liccardo B, Esposito N, Calabrò R. Left ventricular early myocardial dysfunction after chronic misuse of anabolic androgenic steroids: a 
Doppler myocardial and strain imaging analysis. $\mathrm{Br} \mathrm{J}$ Sports Med [Internet]. Marzo 2007 [Acceso 18 de mayo 2016]; 41(3):149-55. Disponible en: http://www.ncbi.nlm.nih.gov/pmc/articles/PMC2465218/

56. Parker MW, Thompson PD. Anabolic-androgenic steroids: worse for the heart than we knew?. Circ Heart Fail [Internet]. Julio 2010 [Acceso 18 de mayo 2016]; 3(4):470-1. Disponible en: http://circheartfailure .ahajournals. org/cgi/pmidlookup?view=long\&pmid=206474 88

57. Baggish AL, Weiner RB, Kanayama G, Hudson JI, Picard MH, Hutter AM, et al. Long-term anabolic-androgenic steroid use is associated with left ventricular dysfunction. Circ Heart Fail [Internet]. Julio 2010 [Acceso 19 de mayo 2016]; 3(4):472-6. Disponible http://www.ncbi.nlm.nih.gov/pmc/articles/PMC2909423/

58. Angell PJ, Chester N, Sculthorpe N, Whyte G, George K, Somauroo J. Performance enhancing drug abuse and cardiovascular risk in athletes: implications for the clinician. Br J Sports Med [Internet]. Noviembre 2012 [Acceso 22 de mayo 2016]; 46 Suppl 1:78-84. Disponible en: http://www.ncbi.nlm.nih.gov/pmc/articles/PMC2909423/

59. Odilon A, Evitom S. Esteroides anabolizantes androgénicos y sus efectos colaterales: una revisión científica crítica. Rev. educ. Fis [Revista en internet]. Octubre-diciembre 2013 [Acceso 22 de mayo 2016]; 24(4): 669-679. Disponible en: http://pesquisa.bvsalud.org/portal/resource/es/lil-711196

60. Hernández M, Guillem JL. Abuso de anabolizantes. Psiq Biol [Revista en internet]. 2005 [Acceso 28 de mayo 2016]; 12(1):18-21. Disponible en: http://www.elsevier.es/es-revista-psiquiatria-biologica-46-articulo-abusoanabolizantes-13071129

61. Kicman AT. Pharmacology of anabolic steroids. Br J Pharmacol [Internet]. Junio 2008 [Acceso 6 de junio 2016]; 154(3):502-21. Disponible en: http://www.ncbi.nlm.nih.gov/pmc/articles/PMC2439524/

62. Kanayama G, Brower KJ, Wood RI, Hudson JI, Pope HG Jr. Anabolicandrogenic steroid dependence: an emerging disorder. Addiction [Internet]. Diciembre 2009 [Acceso 6 de junio 2016]; 104(12):1966-78. Disponible en: http://www.ncbi.nlm.nih.gov/pmc/articles/PMC2780436/

63. Kanayama G, Kean J, Hudson JI, Pope HG Jr. Cognitive deficits in long-term anabolic-androgenic steroid users. Drug Alcohol Depend [Internet]. Junio 2013 [Acceso 6 de junio 2016]; 130(1-3):208-14. Disponible en: http://www.ncbi.nlm.nih.gov/pmc/articles/PMC3608708/

64. Torres O, Covarrubias R, Zamora AL, Torres BM, García M, Martínez FG. Anabolic androgenic steroids induce micronuclei in buccal mucosa cells of bodybuilders. Br J Sports Med [Internet]. Septiembre 2007 [Acceso 6 de junio 2016]; $\quad$ 41(9):592-596.

Disponible

http://www.ncbi.nlm.nih.gov/pmc/articles/PMC2465411/

65. Leifman H, Rehnman C, Sjöblom E, Holgersson S. Anabolic androgenic steroids -use and correlates among gym users- an assessment study using questionnaires and observations at gyms in the Stockholm region. Int $\mathrm{J}$ Environ Res Public Health [Internet]. Julio 2011 [Acceso 6 de junio 2016]; 8(7):265674. Disponible en: http://www.ncbi.nlm.nih.gov/pmc/articles/PMC3155322/

Referencias totales citadas: 65

Referencias citadas correspondientes a la Rev Ib CC Act Fis Dep: 0 